\title{
Algorithm about Ordering the Departing Flights on Multi-Runway
}

\author{
Yu Guo \\ School of North China Electric Power University, Baoding 071000, China
}

Keywords: order departing flights, FCFS algorithm, multi-level feedback queue scheduling algorithm

\begin{abstract}
This paper aims at ordering the departing flights on multi-runway, to alleviate the severe flight delays in our country nowadays. We respectively use FCFS scheduling algorithm and multilevel feedback queue scheduling algorithm, to get the result by the simulation calculation above. Finally, the paper gets some conclusions which may have some reference value on the departing flights.
\end{abstract}

\section{Introduction}

This paper makes research on international topic-- delayed flights, which is committed to finding solutions to our problems of severe flight delay situation. According to statistics on flightstats.com, a famous newspaper in Hong Kong said: there were 10 airports with most severe flight delays, among the international airports, while China accounted for 7. Before this paper, we analyzed the data of more than 100 international airports in June 2013, to verify the severe situation of flight delays in China. Meanwhile, the causes found are divided into the following four categories: Airline, traffic, weather and other reasons (including military, passengers and other reasons). Among them, the impact airline has on flight delays cannot be ignored, including the construction of the runway, airport flights order and so on. Reasonable flights departing order will increase the utilization of the runway, to ease the problem of flight delays. In recent years, how to perform flights departing order has already caused heated discussion.

This paper studies the issue of flights departing with airport capacity fixed, two runways both used for taking off for some time. The model considers the scheduling habits of dispatchers who order the flights on the ground (often ordering flights to someplace on a specific runway). Combing with different departing flights having a minimum safe distance, the paper optimizes the order to leave by a multi-level feedback queue scheduling algorithm, to achieve the purpose of saving time (evenly saving more time than FCTS).

Multi-level feedback queue scheduling algorithm, originally an algorithm for CPU processor, is well mapped to our model. It itself is an optimization of FCFS scheduling algorithm (multiple FCFS). Its mechanism is actually when a queue to be processed with a priority, all remaining queues waiting and prioritize. The beauty of this method is that, as a queue to be processed, the remaining queue priority changed, in accordance with the principle of making total delay time minimize. Or it can be said to be iterative optimization for many times, so that whenever there is a spare runway there is use of flights, which improves the utilization of the runway and saves time.

\section{Departing Flights Ordering Model}

\subsection{Ordering Model}

Suppose there are a number of departing flights during peak hours, usually controllers assigned a number of $N_{1}$ flights taking off from the runway 1, a number of $N_{2}$ flights taking off from the runway 2 fixedly. There are a number of $N_{f}$ flights to be waiting taking off.

Where:

$$
\begin{aligned}
& N_{1} \cup N_{2} \cup N_{f}=N \\
& N_{1} \cap N_{2} \cap N_{f}=\varnothing
\end{aligned} \text { Additional notations follows: }
$$


$E T_{i r}$--the time of flight $i$ planning to taking off on runway $r, r=1,2, \cdots, R$;

$A T_{i r}$--the time of flight $i$ taking off actually on runway $r, r=1,2, \cdots, R$;

$X_{\text {ir }}$--the variable of the flight $i$ to runway $r$;

$y_{i j}$--the variable of the flight $i$ to flight $j$;

$S_{i j}$--the minimum safe distance of the plane $i$ to plane $j$;( unit: min)

Variable description:

$x_{i j}=\left\{\begin{array}{l}1, \text { flight } i \text { assigned to taking off on runway } j \\ 0, \text { others }\end{array}\right.$

$y_{i j}=\left\{\begin{array}{l}1, \text { flight } i, j \text { assigned to the same runway } \\ 0, \text { others }\end{array}\right.$

$z_{i j}=\left\{\begin{array}{l}1, \text { flight } i \text { assigned to taking off on runway } j \text { fixedly } \\ 0, \text { others }\end{array}\right.$

This paper gives the following objective function to get the minimum of total delay time $z=\min \sum_{i=1}^{N}\left\{A T_{i r}-\min \left[E T_{i 1}-E T_{i 2}\right]\right\}$

The followings are kinds of constraints that should be satisfied:

$$
\left\{\begin{array}{lr}
\sum_{r=1}^{R} X_{i r}=1 & \left(i=1,2, \cdots, N_{f}\right) \\
\sum_{r=1}^{N_{1}} z_{i r}=1 & (r=1) \\
\sum_{r=1}^{N_{2}} Z_{i r}=1 & (r=2) \\
A T_{i+1}-A T_{i} \geq S_{i j} &
\end{array}\right.
$$

MATLAB programming, model will find the optimal solution according to the objective function.

\subsection{Case Study}

Assume there are 10 flights waiting to take off during a period of busy time at airport. There are two parallel runways at the airport. We define that a period of busy time is 15 minutes. Suppose there are 10 flights taking off within 15 minutes. Usually controllers assign flight CES2295, CSC8801 taking off from the runway 1, flight CES 2305 taking off from the runway 2 fixedly. According to the ICAO, the plane can be sorted into heavy $(H)$, large $(L)$ and small $(S)$. The minimum safe distance (Here use time to take place of distance)between two planes is shown in Table 1.

\begin{tabular}{|c|c|c|c|}
\hline \multicolumn{3}{|c|}{ Table1. minimum safe time between departing } \\
flight \\
\hline \multirow{2}{*}{$\begin{array}{c}\text { The former plane } \\
i\end{array}$} & \multicolumn{3}{|c|}{$S_{i j}$} \\
\cline { 2 - 4 } & plane $j H$ & plane $_{j L}$ & $\begin{array}{c}\text { plane } \\
j S\end{array}$ \\
\hline H & 2 & 2 & 3 \\
\hline L & 2 & 2 & 2 \\
\hline S & 2 & 2 & 2 \\
\hline
\end{tabular}

Suppose we have known each flight's time of planning to take off, shown as table 2. 


\begin{tabular}{cccccccccccc}
\hline \multicolumn{10}{c}{ Table 2. Each flight's time of planning to take off } \\
\hline \multirow{2}{*}{ runway } & CHH & CES & CES & CSC & CES & CES & OKA & CCA & CSC & CES \\
& 7855 & 2342 & 2295 & 8801 & 2161 & 2305 & 2851 & 4184 & 8843 & 2939 \\
\hline 1 & 11 & 15 & 6 & 6 & 9 & 7 & 15 & 6 & 6 & 9 \\
\hline 2 & 10 & 17 & 7 & 7 & 12 & 6 & 17 & 7 & 7 & 12 \\
\hline $\begin{array}{c}\text { Plane } \\
\text { sort }\end{array}$ & H & L & H & L & S & H & H & H & H & S \\
\hline
\end{tabular}

When using FCFS algorithm (a first come first served principle), the calculation is:

Table 3. using FCFS algorithm with two runways

\begin{tabular}{|c|c|c|c|c|}
\hline Flight & $\begin{array}{l}\text { Predicted } \\
\text { taking-off } \\
\text { time/min }\end{array}$ & $\begin{array}{l}\text { Runway } \\
\text { number }\end{array}$ & $\begin{array}{l}\text { Actually } \\
\text { taking- } \\
\text { off } \\
\text { time/min }\end{array}$ & $\begin{array}{r}\text { Delay } \\
\text { time/min }\end{array}$ \\
\hline
\end{tabular}

\begin{tabular}{ccccc}
\hline CES2295 & 6 & 1 & 6 & 0 \\
\hline CSC8801 & 6 & 1 & 8 & 2 \\
\hline CCA4184 & 6 & 1 & 10 & 4 \\
\hline CSC8843 & 6 & 1 & 12 & 6 \\
\hline CES2305 & 6 & 2 & 6 & 0 \\
\hline CES2161 & 9 & 1 & 15 & 6 \\
\hline CES2939 & 9 & 1 & 17 & 8 \\
\hline CHH7855 & 10 & 2 & 10 & 0 \\
\hline CES2342 & 15 & 1 & 19 & 4 \\
\hline OKA2851 & 15 & 1 & 21 & 6 \\
\hline
\end{tabular}

The total delay time with FCFS optimization algorithm is to be 36 minutes.

Due to FCFS algorithm relatively low, we use a multi-level feedback queue scheduling algorithm to optimize the model, an optimization of FCFS scheduling algorithm (that is multiple FCFS). With a queue to be processed, in accordance with the minimum of the total delay time, the remaining priority queue changed. After repeatedly iterative optimization, the results are as follows: 


\begin{tabular}{crrrr}
\hline \multicolumn{5}{c}{ Table 4. use a multi-level feedback queue scheduling } \\
algorithm with two runways \\
\hline Flight & $\begin{array}{c}\text { Predicted } \\
\text { taking-off } \\
\text { time/min }\end{array}$ & $\begin{array}{c}\text { Runway } \\
\text { number }\end{array}$ & $\begin{array}{c}\text { Actually } \\
\text { taking- } \\
\text { off } \\
\text { time/min }\end{array}$ & $\begin{array}{c}\text { Delay } \\
\text { time/min }\end{array}$ \\
\hline CES2295 & 6 & 1 & 6 & 0 \\
\hline CSC8801 & 6 & 1 & 8 & 2 \\
\hline CCA4184 & 6 & 2 & 6 & 0 \\
\hline CSC8843 & 7 & 2 & 8 & 1 \\
\hline CES2305 & 6 & 1 & 10 & 4 \\
\hline CES2161 & 11 & 1 & 12 & 1 \\
\hline CES2939 & 9 & 1 & 15 & 6 \\
\hline CHH7855 & 12 & 2 & 10 & -2 \\
\hline CES2342 & 17 & 2 & 12 & -5 \\
\hline OKA2851 & 17 & 2 & 14 & -3 \\
\hline
\end{tabular}

Where :

In some conditions, the flight delay time is negative. When a plane is allowed to take off, we let the flight with negative delay time to leave first, which could not participate in ordering .The rest left is in the given order..

The total delay time of multi-level feedback queue scheduling algorithm is calculated to be 14 minutes. Compared to the principle of first come first served (FCFS algorithm), the total delay rate is sorted by nearly $60 \%$, which obviously reduces flights delay time, together with improving the utilization of the runway.

\section{Summary}

Flight ordering problem is what our current aviation staff should concentrate on, adopting rational optimization when assigning runway can effectively improve space utilization of airspace. The paper is majored in the problem of delayed flights. It gives the optimized flight ordering solutions. Using respectively FCFS scheduling algorithm and multilevel feedback queue scheduling algorithm, we study the case by MATLAB simulation. The results show that the multi-level feedback queue scheduling algorithm saves more delay time than FCFS scheduling algorithm (the total delay time will be reduced by more than half). Based on the actual situation provided by references, the paper shows certain algorithms, hoping to have some reference value on departing flights ordering.

\section{References}

[1]. Wei Yun, Wang Lili, et al. Departure flights sort based on control diet a multi-runway airport. Aviation Computing Technology. 2015, 45(1): 45-47.

[2]. Zhao Guodong, et al. Approach and departure flight scheduling problems on multiple runways. Technology and Enterprise. 2016 (2): 21-21.

[3]. Mathematical modeling algorithms and application exercises Answers [M]. National Defense Industry Press, 2013. 